\title{
Appetitive-aversion interactions: Facilitation of aversive conditioning by prior appetitive training in the rat
}

\author{
ANTHONY DICKINSON \\ University of Sussex, Brighton, England BNI $9 Q G$
}

\begin{abstract}
In Experiment 1, four groups of rats received conditioned suppression training in which a tone was reinforced with shock. If the tone had been previously paired with response-independent food, aversive conditioning was slightly facilitated by comparison to control groups preexposed either to the tone randomly associated with food or to the tone and food unpaired. However, by comparison to a control which was not preexposed to the tone, animals receiving prior pairings of the tone and food showed retarded aversive conditioning. Experiment 2 replicated the facilitation in aversive conditioning after the tone had been paired with food relative to the random control condition and demonstrated that this difference occurred even if the tone and background stimuli continued to be associated with response-independent food during aversive conditioning. This result suggests that pairing a stimulus with an appetitive reinforcer reduces the retardation of aversive conditioning produced by stimulus preexposure.
\end{abstract}

There is good evidence that appetitive and aversive Pavlovian conditioning cannot be treated independently. In fact, a number of theorists (e.g., Bindra, 1974; Estes, 1969; Gray, 1975; Konorski, 1967; Millenson \& de Villiers, 1972; Rescorla \& Solomon, 1967; Stein, 1964) have argued that appetitive and aversive response systems are mutually antagonistic. In agreement with this idea, it is fairly well established that aversive Pavlovian conditioning retards the subsequent development of an appetitive Pavlovian conditioned response to the same conditioned stimulus (Konorski \& Szwejkowska, 1956; Scavio, 1974). By contrast, the outcome of the opposite transfer paradigm which investigates the effect of prior appetitive Pavlovian training on aversive conditioning with the same conditioned stimulus (CS) is less well determined.

Konorski and Szwejkowska (1956) failed to establish a stable leg flexion conditioned response in one dog by pairing a metronome with a shock if this stimulus had been previously turned into a CS for salivation by associating it with food. However, Jackson (1974) was unable to detect a comparable retardation effect using rats in a conditioned suppression procedure. Pairing an auditory CS with response-independent food did not significantly alter the rate at which suppression developed when the same CS was subsequently associated with shock.

This research was supported by a grant from the United Kingdom Science Research Council. Requests for reprints should be sent to Anthony Dickinson, Laboratory of Experimental Psychology, University of Sussex, Brighton, England BN1 9QG.
Jackson (1974) employed a relatively long CS ( 2 min) and administered only 12 CS presentations during appetitive conditioning. The purpose of the first experiment was to investigate the effect of pairing a CS with response-independent food on the acquisition of conditioned suppression after more extended appetitive conditioning with a shorter CS.

\section{EXPERIMENT 1}

\section{Subjects}

Method

The subjects were 32 experimentally naive male hooded Lister rats. Before training, they were randomly assigned to four equal groups and gradually reduced $80 \%$ of their free-feeding weight. Thereafter, they were maintained at this level by being given a restricted amount of food after each session.

\footnotetext{
Apparatus

Training was conducted in four similar operant chambers $(26 \times 26 \times 20 \mathrm{~cm})$ housed in sound- and light-resistant shells. Each chamber was equipped with a Campden Instrument rat lever, modified to be retractable, and an adjacent recessed food magazine with a Perspex flap door that operated a microswitch. Food reinforcement consisted of the delivery of a single $45-\mathrm{mg}$ pellet (Campden Instruments Ltd.) and was accompanied by the operation of two heavy-duty relays to signal delivery. The grid floor was made of .6-cm-diam stainless steel rods spaced $1.6 \mathrm{~cm}$ center to center and arranged to lie parallel to the front wall. Shocks of $.5-\mathrm{sec}$ duration could be delivered to the grid floor and lever by a Campden Instruments shock source (No. 512). This shock source gives a constant current squarewave output with a $47 \%$ duty cycle. The intensity was set to give a peak output current of $.5 \mathrm{~mA}$. The shock was delivered by a Campden Instruments shock scrambler (No. 521S), which runs at $25 \mathrm{~Hz}$ with eight separate states per cycle. The CS was a 30-sec, 3,000-Hz tone generated by a Campden Instruments audio generator (No. 258) and delivered from a speaker mounted in the front wall above the magazine. The CS intensity was approximately $85 \mathrm{~dB}$ (against a background level of $75 \mathrm{~dB}$ re: $\left.20 \mu \mathrm{N} / \mathrm{m}^{2}\right)$.
} 


\section{Procedure}

After magazine and continuously reinforced leverpress training, responses were reinforced on a variable interval (VI) 30-sec schedule and a VI 1-min schedule during the next two sessions. In all subsequent sessions, responding was reinforced on a VI 2-min schedule. The sessions started and terminated with insertion and retraction of the lever, respectively, and were $50 \mathrm{~min}$ long throughout the experiment.

Appetitive training. All rats received eight sessions of appetitive training, during which responding continued to be reinforced on a VI 2-min schedule. Animals in groups $P$ (paired), $\mathrm{R}$ (random), and $\mathrm{U}$ (unpaired) received $20 \mathrm{CS}$ presentations per session with an intertrial interval (ITI) of 2 min. For rats in group $P$, food pellets were delivered independently of responding on a fixed time (FT) 12-sec schedule during each CS presentation. Rats in groups $R$ and $U$ also received responseindependent food on the same FT 12-sec schedule during 20 30 -sec periods in each session. For group $\mathbf{R}$ these periods were selected randomly from the 10030 -sec periods comprising the whole session, while for group $U$ the periods were selected randomly from the 60 periods remaining when the $20 \mathrm{CS}$ and immediately succeeding 30-sec periods were excluded. As a result, animals in group $U$ never received response-independent food during or immediately following a CS presentation. The rats in the final group, group F (food-alone), were not exposed to the CS but received response-independent food during the same periods as group P. In order to prevent the accumulation of pellets in the magazine which might not be eaten until after the termination of the appropriate stimulus condition, programmed pellets were not delivered unless the magazine flap had been operated since the last pellet delivery.

Aversive training. During each of two aversive training sessions, two CS presentations terminating in a shock were given to all animals. The first trial occurred $19.5 \mathrm{~min}$ after the start of the session, and the ITI was $24.5 \mathrm{~min}$.

Response suppression during a trial was expressed as a suppression ratio to attenuate the effect of individual differences in the overall rate of responding. The ratio had the form $\mathbf{A} /(\mathbf{A}+\mathbf{B})$. During appetitive training $\mathbf{A}$ represented the rate of responding during the $C S$ for groups $P, R$, and $U$, and the rate during the equivalent periods for group $F$, while during aversive training, $A$ represented the rate during the $C S$ for all groups. In all cases, B was based on the rate of responding during 1 -min periods immediately preceding each trial. The absence of suppression is indicated by a ratio of .50 , and maximum suppression by a ratio of zero.

\section{Results and Discussion}

One subject from each group had to be excluded from the analysis and presentation of the results owing to a recording failure associated with one of the chambers during aversive training.

Figure 1 illustrates the mean suppression ratios of the various groups during both appetitive and aversive training. During appetitive training, both group $\mathbf{P}$ and group $\mathbf{F}$ showed some suppression with the paired condition producing more suppression. Groups $R$ and $U$ remained unsuppressed. With continued training, the differences between the groups tended to decrease. Statistical analysis of suppression ratios during appetitive training revealed a significant effect of groups $(F=13.47$, df $=3 / 24$, $\mathrm{p}<.01$ ) and a significant Group by Trial Block interaction $(F=3.42, \mathrm{df}=21 / 168, \mathrm{p}<.01)$. Orthogonal a posteriori comparisons of the group mean suppression ratios averaged over all appetitive

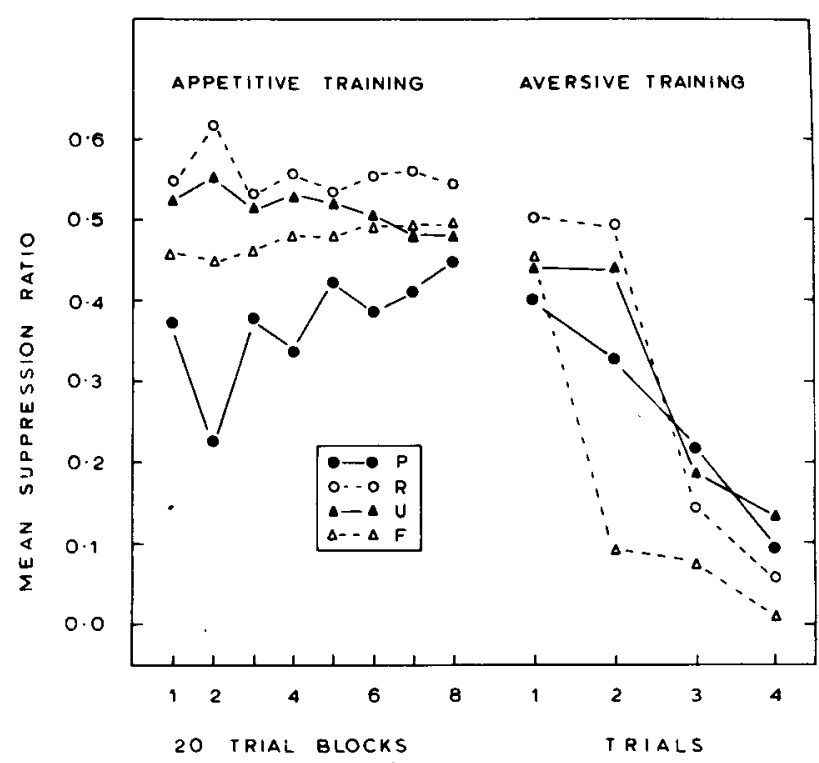

Figure 1. Experiment 1: Group mean suppression ratios during appetitive and aversive training. The ratios are displayed for 20-trial blocks during appetitive training and for single trials during aversive training. $P$, paired; $R$, random; $U$, unpaired; F, food-alone.

training sessions showed that while groups $\mathbf{R}$ and $\mathbf{U}$ did not differ $(F<1)$, group $P$ was significantly more suppressed than groups $R$ and $U$ combined $(\mathrm{F}=12.84$, df $=3 / 24, \mathrm{p}<.01)$. The comparison of group $F$ with groups $P, R$, and $U$ combined was not significant $(F<1)$, indicating that the suppression ratios for group $F$ lay between those of group $P$ and those of groups $R$ and $U$. There was no significant difference between the pretrial response rates of the various groups $(F<1)$.

The greater suppression shown by group $P$ relative to group $F$ indicates that the suppression produced by the CS in group $P$ was only partially attributable to the fact that animals in the paired condition had less time available for responding as they were consuming the response-independent food pellets. Since it is commonly found that presentation of an appetitive CS suppresses positively reinforced baseline responding (e.g., Azrin \& Hake, 1969; Miczek \& Grossman, 1971), these results provide independent evidence that the tone had become an appetitive CS in group $P$ prior to aversive training.

Although the groups did not differ significantly in suppression on the first aversive training trial, group $F$ developed suppression more rapidly than groups $R$ and $U$, with the difference being most marked on the second trial. Prior appetitive conditioning to the CS in group $P$ retarded conditioning with respect to a non-preexposed condition (group F) but, if anything, facilitated conditioning on the second trial by comparison to the preexposed conditions (groups $R$ and $U$ ). Separate analyses of suppression ratios on each aversive training trial showed 
a significant effect of appetitive training on Trial 2 $(\mathrm{F}=6.55, \mathrm{df}=3 / 24, \mathrm{p}<.01)$ but not on Trials 1 , 3 , and 4 ( $F<1.37$, df $=3 / 24, p>.25$, in all cases). Orthogonal comparisons of the mean suppression ratios on Trial 2 revealed that groups $R$ and $U$ did not differ $(F<1)$ but that group $F$ was significantly more suppressed than groups $R$ and $U$ combined $(F=5.27, \mathrm{df}=3 / 24, \mathrm{p}<.01)$. The fact that group $P$ did not differ significantly from groups $F$, $R$, and $U$ combined $(F<1)$ confirms the impression from the graphic data that the suppression ratios for group $P$ lay between those of group $F$ and groups $R$ and $U$. An analysis of a pretrial response rates showed that both the effect of Groups $(F<1)$ and the Group by Trial interaction $(F=1.14$, df $=18 / 72, p>.25$ ) failed to reach significance.

The results of this experiment provide no support for the idea that pairing a CS with an appetitive reinforcer retards subsequent aversive conditioning over and above the effect due simply to CS preexposure. In fact, appetitive pretraining appeared to reduce the magnitude of the retardation effect resulting from preexposure.

\section{EXPERIMENT 2}

There are at least two possible reasons why a retardation effect was not observed in the paired condition of Experiment 1. First, during aversive training, the CS was presented in the absence of response-independent food so that any appetitive conditioning may have extinguished before retardation could be observed. Second, there are grounds for believing that retardation might occur only if an association is maintained between the background stimuli and the appetitive reinforcer during aversive training. In the only successful demonstration of retarded aversive conditioning, Konorski and Szwejkowska (1956) gave a single aversive conditioning trial to the relevant CS per session intermixed with a number of appetitive conditioning trials to other CSs. When the frequency of aversive conditioning trials was increased, a stable defensive conditioned response was established.

In order to investigate the possible importance of these factors, animals in the second experiment were given either paired or random appetitive pretraining. During aversive conditioning, half the animals in each condition experienced pairings of the CS and shock in the absence of response-independent food while the remaining rats received free food during both the CS and the intertrial intervals. This later procedure should maintain the association of both the CS and the background stimuli with the appetitive reinforcer during aversive training.

Subjects and Apparatus

The subjects were 32 experimentally naive male hooded
Lister rats. Before the start of training, they were randomly assigned to two equal groups. The deprivation regime, apparatus, stimuli, and reinforcers were identical to those employed in Experiment $\mathbf{I}$.

\section{Procedure}

After the leverpress response was established with the VI 2-min schedule using the procedure employed in Experiment 1, all animals received eight 50 -min appetitive training sessions. Rats in the paired $(P)$ and random $(R)$ conditions received identical training to the comparable groups of Experiment 1 . In the paired condition, the CS was associated with responseindependent food while animals in the random condition experienced no consistent relationship between the CS and free food.

Following appetitive training, two aversive conditioning sessions, each containing two trials, were given. For half the animals in the paired and random conditions (groups $P$ and $R$ respectively), response-independent food was not delivered during aversive training and the aversive conditioning procedure was identical to that employed in Experiment 1. The remaining animals in the paired and random conditions (groups PF and RF) received the same aversive conditioning procedure except that the presentation of response-independent food was continued. In order to maintain the association of the CS with the appetitive reinforcer, both groups $P F$ and $R F$ received free food on the FT 12-sec schedule during each CS. In addition, on each session, free food was also delivered during 18 30-sec non-CS periods which occurred before the first trial and during the ITI. The interval between these periods of free food was $2 \mathrm{~min}$. The delivery of food outside CS trials should maintain an association between the background stimuli and the appetitive reinforcer. Response suppression was expressed in terms of the same ratio as employed in Experiment 1 except that the pretrial response rate was based on responding during $30-\mathrm{sec}$ periods preceding each trial.

\section{Results and Discussion}

Figure 2 illustrates the mean suppression ratios in the various conditions during both appetitive and aversive training. As in Experiment 1, the paired condition produced more suppression than the random condition during appetitive training $(F=106.49, \mathrm{df}=1 / 30, \mathrm{p}<.01)$. The pretrial response rates in the two conditions did not differ $(\mathrm{F}=1.08, \mathrm{df}=1 / 30, \mathrm{p}>.25)$.

As the development of suppression during aversive training was slower than in Experiment 1, suppression ratios were calculated on the basis of the number of responses cumulated over pairs of trials in order to increase the stability of the ratios. During aversive training, animals receiving responseindependent food (groups PF and RF) showed more suppression than the groups without free food (groups $\mathbf{P}$ and $\mathbf{F}$ ). The results of Experiment 1 suggest that this difference was at least partially due to the time spent consuming the food pellets. More significantly, the results show that whether or not animals received free food during aversive training prior pairings of the CS with food speeded the acquisition of suppression even though the level of suppression on the first two-trial block did not vary as a function of the appetitive training condition. A separate analysis of suppression on each two-trial block showed a significant effect of delivering free 


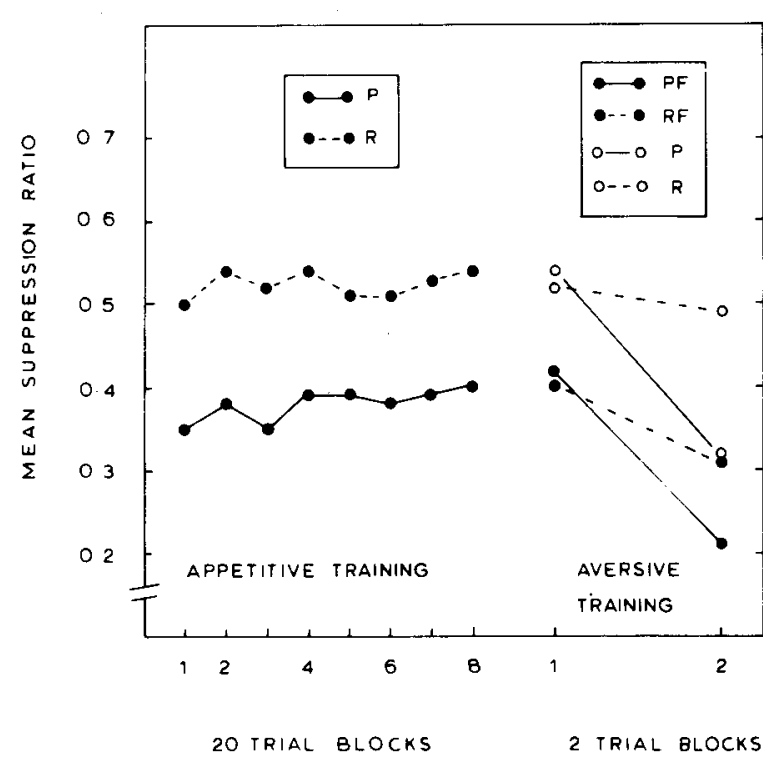

Figure 2. Experiment 2: Group mean suppression ratios during appetitive and aversive training. The ratios are displayed for 20-trial blocks during appetitive training and 2-trial blocks during aversive training. Appetitive training: $\mathbf{P}$, paired; $\mathbf{R}$, random. Aversive training: PF, paired with free food; RF, random with free food; $P$, paired without free food; $R$, random without free food.

food on both the first $(F=11.39, \mathrm{df}=1 / 28$, $\mathrm{p}<.01)$ and second block $(\mathrm{F}=8.78, \mathrm{df}=1 / 28$, $p<.01)$. The effect of the appetitive training contingency (paired vs. random) was significant on the second $(F=7.23$, df $=1 / 28, p<.02)$ but not the first trial block $(F<1)$. Neither the appetitive training contingency $(F=2.54$, df $=1 / 28, p>.10)$ nor the delivery of free food $(F<1)$ affected the pretrial response rates during aversive conditioning.

These results essentially confirm the findings of Experiment 1 by showing that pairing a CS with an appetitive reinforcer facilitates subsequent aversive conditioning by comparison to a control group receiving similar preexposure to the $\mathrm{CS}$. The magnitude of this facilitation appears to be unaffected by whether the association of the CS and the background stimuli with the appetitive reinforcer is maintained during aversive conditioning.

\section{GENERAL DISCUSSION}

These experiments provide little support for the idea that the mechanisms mediating appetitive Pavlovian conditioning exert an inhibitory influence on the elaboration of aversive Pavlovian conditioning. In fact, appetitive training appears to facilitate subsequent aversive conditioning by comparison to preexposed controls. This finding is in line with the results of a number of studies showing that the use of an appetitive Pavlovian CS as a warning signal can facilitate the acquisition of a discrete-trial avoidance response (Bacon \& Bindra, 1967; Braud, 1971; Overmier \& Payne, 1971). Of course, it is possible that the mechanisms producing this facilitation mask the emergence of an inhibitory interaction, but without further experimental analysis this idea cannot be evaluated.

As already noted, a number of experiments have shown that the superimposition of an appetitive Pavlovian CS on a positively reinforced baseline can produce suppression, a finding confirmed in the first experiment. However, it is unlikely that the facilitation seen in the paired condition was due to the addition of suppression formed by pairing the CS with food to that produced by the aversive reinforcer. In neither experiment did the level of suppression maintained by the paired and control groups differ on the initial aversive conditioning trial.

Fowler, Fago, Domber, and Hochhauser (1973) and Overmier and Bull (1970) have both suggested that the facilitation seen in a variety of acrossreinforcer transfer studies is best understood by assuming that pairing a CS with a given reinforcer endows it with general signaling or cueing properties. This signaling capacity then enhances the associability of that CS with another type of reinforcer. However, as the paired group of Experiment 1 actually showed retarded aversive acquisition by comparison to a non-preexposed group, there is little justification for employing such an explanation in the present situation.

An alternative account assumes that the facilitation seen in the paired condition reflects a reduction in the factor retarding conditioning in the preexposed control groups. The difference between the rate of aversive conditioning shown by the control group not preexposed to the CS in Experiment 1, group $F$, and the preexposed control groups, groups $\mathbf{R}$ and $\mathrm{U}$, represents an example of latent inhibition (cf. Lubow, 1973). Within this context, it appears that pairing a CS with food reduces the development of latent inhibition when measured using an aversive reinforcer. Such an explanation is in line with Lubow, Schnur, and Rifkin's (1976) recent demonstration that a number of procedures likely to maintain attention to a CS reduce latent inhibition.

\section{REFERENCES}

Azrin, N. H., \& Hake, D. F. Positive conditioned suppression: Conditioned suppression using positive reinforcers as the unconditioned stimuli. Journal of the Experimental Analysis of Behavior, 1969, 12, 167-173.

BACON, W. E., \& Bindra, D. The generality of the incentivemotivational effects of classically conditioned stimuli on instrumental learning. Acta Biologiae Experimentalis, 1967, 27, 185-197.

BindRA, D. A motivational view of learning, performance, and behavior modification. Psychological Review, 1974, 81, 199-213. 
BRAUD, W. G. Effectiveness of "neutral," habituated, shockrelated, and food-related stimuli as CSs for avoidance learning in gold fish. Conditional Reflex, 1971, 6, 153-156.

Estes, W. K. Outline of a theory of punishment. In R. M. Church \& B. A. Campbell (Eds.), Punishment and aversive behavior. New York: Appleton-Century-Crofts, 1969.

Fowler, H., FAgo, G. C., Domber, E. A., \& Hochhauser, M. Signaling and affective functions in Pavlovian conditioning. Animal Learning \& Behavior, 1973, 1, 81-89.

GRAY, J. A. Elements of a two process theory of leaming. London: Academic Press, 1975.

JACKSON, D. E. CS-free food contingencies and subsequent acquisition of conditioned suppression: No transfer effect. Bulletin of the Psychonomic Society, 1974, 4, 235-236.

KONORSKI, J. Integrative activity of the brain. Chicago: University of Chicago Press, 1967.

KonORSKI, J., \& Szwejrowsra, G. Reciprocal transformation of heterogeneous conditioned reflexes. Acta Biologiae Experimentalis, 1956, 17, 141-165.

Lubow, R. W. Latent inhibition. Psychological Bulletin, 1973, 79, 398-407.

Lubow, R. E., Schnur, P., \& RifkIN, B. Latent inhibition and conditioned attention theory. Journal of Experimental Psychology: Animal Behavior Processes, 1976, 2, 163-174.

Miczek, K. A., \& Grossman, S. P. Positive conditioned suppression: Effects of CS duration. Journal of the Experimental Anal- ysis of Behavior, 1971, 15, 243-247.

Millenson, J. R., \& DE Villiers, P. A. Motivational properties of conditioned anxiety. In R. M. Gilbert \& J. R. Millenson (Eds.), Reinforcement: Behavioural anabyses. New York: Academic Press, 1972.

OVERMIER, J. B., \& BulL, J. A., III. Influence of appetitive Pavlovian conditioning upon avoidance behavior. In J. $\mathbf{H}$. Reynierse (Ed.), Current issues in animal learning. Lincoln: University of Nebraska Press, 1970.

Overmier, J. B., \& PAYne, R. J. Facilitation of instrumental avoidance learning by prior appetitive Pavlovian conditioning to the cue. Acta Neurobiologiae Experimentalis, 1971, 31, 341-349.

Rescorla, R. A., \& Solomon, R. L. Two-process learning theory: Relationship between Pavlovian conditioning and instrumental learning. Psychological Review, 1967, 74, 151-182.

ScAvio, M. J. Classical-classical transfer: Effects of prior aversive conditioning upon appetitive conditioning in rabbits (Oryctolagus cunicullus). Journal of Comparative and Physiological Psychology, 1974, 86, 107-115.

STEIN, L. Reciprocal action of reward and punishment mechanisms. In R. G. Heath (Ed.), The role of pleasure in behavior. New York: Harper \& Row, 1964.

(Received for publication November 21, 1975; revision received July 7,1976 .) 